\title{
The structure of the proton: The CT14 QCD global analysis
}

\author{
S. Dulat ${ }^{1,5}$, T.-J. Hou ${ }^{2}$, J. Gao ${ }^{3}$, M. Guzzi ${ }^{4, a}$ J. Huston ${ }^{5}$, P. Nadolsky², J. Pumplin ${ }^{5}$, C. Schmidt ${ }^{5}$, \\ D. Stump ${ }^{5}$, and C.-P. Yuan ${ }^{5}$ \\ ${ }^{1}$ School of Physics Science and Technology, Xinjiang University, Urumqi, Xinjiang 830046 China. \\ ${ }^{2}$ Department of Physics, Southern Methodist University, Dallas, TX 75275-0181, U.S.A. \\ ${ }^{3}$ High Energy Physics Division, Argonne National Laboratory, Argonne, Illinois 60439, U.S.A. \\ ${ }^{4}$ The University of Manchester, Oxford Road, M13 9PL Manchester, United Kingdom. \\ ${ }^{5}$ Department of Physics and Astronomy, Michigan State University, East Lansing, MI 48824 U.S.A.
}

\begin{abstract}
A brief description of the CT14 global analysis of quantum chromodynamics at next-to-next-to leading order (NNLO) from the CTEQ-TEA group is given. Predictions for standard candle processes at the LHC are illustrated together with the main features of the CT14 parton distribution functions.
\end{abstract}

\section{Introduction}

Understanding of the structure of the proton is crucial to a multitude of high-energy physics programs at the Large Hadron Collider (LHC). Interpretation of experimental measurements at hadron colliders relies on precise knowledge of fundamental QCD parameters and distribution of proton's constituent quarks and gluons: the parton distribution functions (PDFs). The LHC Run-1 culminated in the discovery of the Higgs boson [1,2]. The LHC Run-2 will perform measurements of the Higgs boson properties and of various electroweak (EW) observables with extraordinary accuracy in new kinematic regimes. For both precision measurements and discovery of possible new physics, it is important to have the proper tools for the calculation of the relevant cross sections. These tools include both matrix element determinations at higher orders in perturbative QCD and EW theory, and precision PDFs.

The CT10 parton distribution functions were published at next-to-leading order (NLO) in 2010 [3], followed by the CT10 next-to-next-to leading order (NNLO) parton distribution functions in 2013 [4]. These PDF ensembles were determined using diverse experimental data from fixed-target experiments, HERA and the Tevatron collider, but without data from the LHC. The new CT14 global analysis [5] includes data from the LHC for the first time, as well as updated data from the Tevatron and from HERA experiments. Various CT14 PDF sets have been produced at the leading order (LO), NLO and NNLO and are available from LHAPDF [6].

Features of the CT14 analysis. The CT14 PDFs are determined from data on inclusive highmomentum transfer processes, for which perturbative QCD is expected to be reliable. For example, in the case of deep inelastic lepton scattering, only data with $Q>2 \mathrm{GeV}$ and $W>3.5 \mathrm{GeV}$ are used. Data in this region are expected to be relatively free of non-perturbative effects, such as higher twists or nuclear corrections.

${ }^{\text {a} P r e s e n t e r, ~ e-m a i l: ~ m a r c o . g u z z i @ m a n c h e s t e r . a c . u k ~}$ 
For the majority of processes in the CT14 fit, theoretical predictions are included at the NNLO level of accuracy. In particular, a NNLO treatment [7] of heavy-quark mass effects in neutral-current DIS is realized in the ACOT- $\chi$ scheme [8-10] and is essential for obtaining correct predictions for LHC electroweak cross sections [11, 12]. Measurements for charged-current DIS and inclusive jet production represent the only exception to this rule and are included at NLO only. In both cases, the complete NNLO contributions are not yet available, but it can be argued based on some investigations that the expected effect of missing NNLO effects is small relatively to current experimental errors (see Sec.II in Ref. [5]).

The new LHC measurements [13-15] of $W / Z$ cross sections directly probe flavor separation of $u$ and $d$ (anti-)quarks in an $x$-range around 0.01 that was not directly assessed by the previously available experiments. The updated measurements of electron charge asymmetry from the DØ collaboration [16] included in the CT14 analysis probe the $d$ quark PDF at $x>0.1$. To better estimate variations in relevant PDF combinations, such as $d(x, Q) / u(x, Q)$ and $\bar{u}(x, Q) / \bar{d}(x, Q)$, the number of free PDF parameters is increased to 28, compared to 25 in CT10 NNLO.

As another important modification, CT14 employs a novel flexible parametrization for the PDFs, based on the use of Bernstein polynomials (reviewed in the Appendix of Ref. [5]). The shape of the Bernstein polynomials is such that a single polynomial is dominant in each given $x$ range, reducing undesirable correlations among the PDF parameters that sometimes occurred in CT10. In the asymptotic limits of $x \rightarrow 0$ or $x \rightarrow 1$, the new parametrization forms allow for the possibility of arbitrary constant ratios of $d / u$ or $\bar{d} / \bar{u}$, in contrast to the more constrained behavior assumed in CT10.

The CT14 PDF error sets is obtained using two techniques, the Hessian method [17] and MonteCarlo sampling [18]. Lagrange multiplier studies [19] have also been used to verify the Hessian uncertainties, especially in regions not well constrained by data. This applies at NNLO and NLO; no error sets are provided at $\mathrm{LO}$ due to the difficulty of defining meaningful uncertainties at that order.

A central value of $\alpha_{s}\left(M_{Z}\right)$ of 0.118 has been assumed in the global fits at NLO and NNLO, but PDF sets at alternative values of $\alpha_{s}\left(m_{Z}\right)$ are also provided. CT14 prefers $\alpha_{s}\left(M_{Z}\right)=0.115_{-0.004}^{+0.006}$ at NNLO $(0.117 \pm 0.005$ at NLO) at $90 \%$ confidence level (C.L.). These uncertainties from the global QCD fits are larger than those of the data from LEP and other experiments included into the world average [20]. Thus, the central PDF sets are obtained using the value of 0.118 , which is consistent with the world average value and was recommended by the PDF4LHC group [21]. The CT14LO PDFs are provided by supplying two versions, one with a 1-loop $\alpha_{s}\left(M_{Z}\right)$ value of 0.130 , and the other with a 2-loop $\alpha_{s}\left(M_{Z}\right)$ value of 0.118 .

\section{Results}

The CT14 fit has a total of 2947 data points included from 33 experiments, producing $\chi^{2}=3252$ at the best fit (with $\chi^{2} / N_{p t}=1.10$ ). The flavor composition of CT14 PDFs has changed somewhat compared to CT10 due to the inclusion of new LHC and Tevatron data sets and to the use of modified parametrization forms. The new PDFs are largely compatible with CT10 within the estimated PDF uncertainty. In Fig. 1 one observes that the CT14 NNLO PDFs have a softer strange-quark distribution at low $x$ and a somewhat softer gluon at high $x$, compared to CT10 NNLO. The CT14 $d$-quark has increased by $5 \%$ at $x \approx 0.05$, after ATLAS and CMS $W / Z$ production data sets at $7 \mathrm{TeV}$ were included. At $x \gtrsim 0.1$, the update of the $\mathrm{D} \varnothing$ charge asymmetry data set in the electron channel has reduced the magnitude of the $d$ quark PDFs by a large amount, and has moderately increased the $u(x, Q)$ distribution. The $d / u$ ratio in Fig. 2 has decreased at high $x$ in comparison to CT10, as a consequence of replacing the $2008 \mathrm{D} \varnothing$ electron charge asymmetry $\left(0.75 \mathrm{fb}^{-1}\right.$ [22]) measurement by the new $9.7 \mathrm{fb}^{-1}$ data set [16]. The $d / u$ ratio approaches a constant value in the $x \rightarrow 1$ limit due to the 

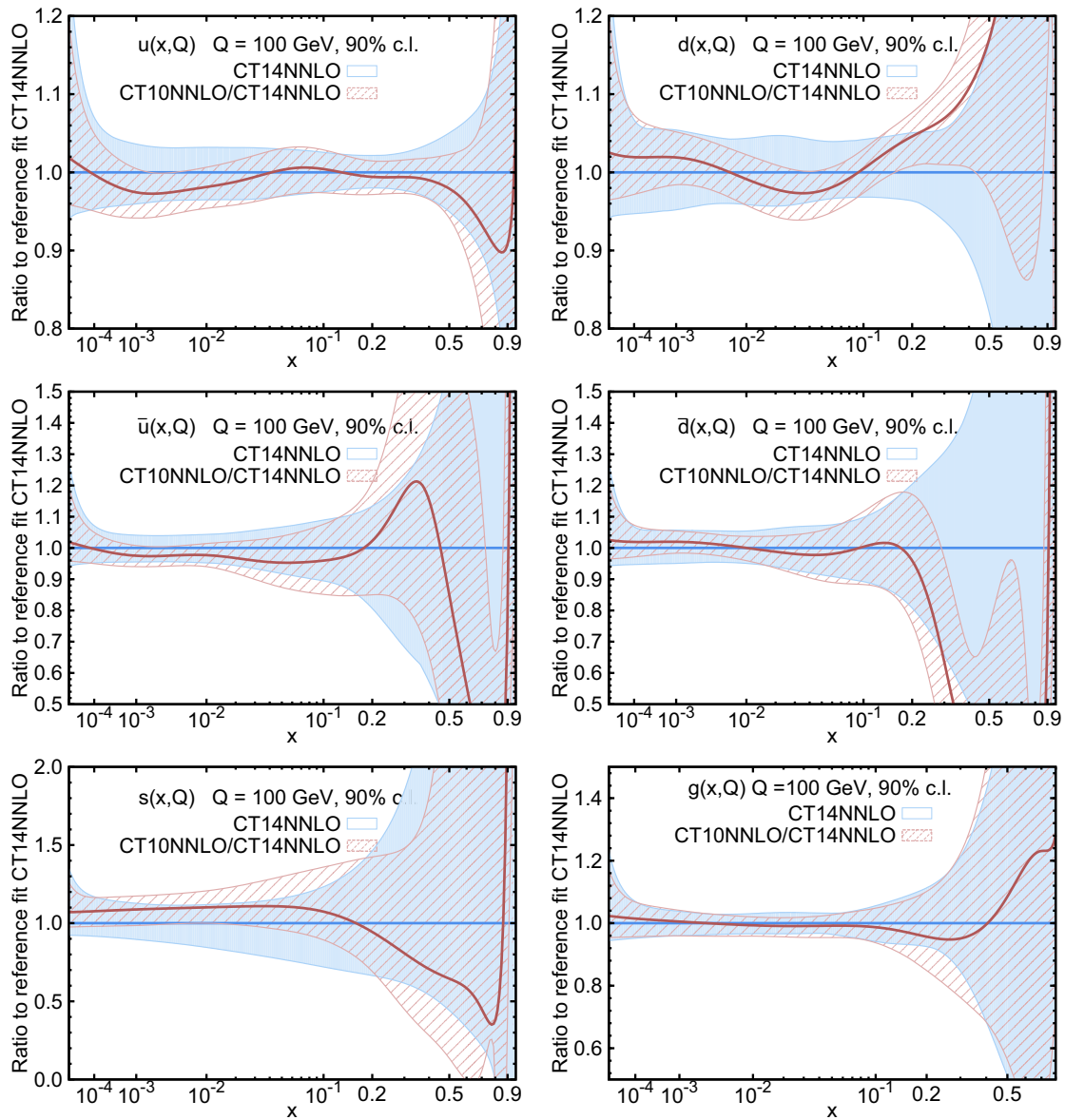

Figure 1. Comparison of 90\% C.L. PDF uncertainties from CT14 NNLO (solid blue) and CT10 NNLO (red dashed) error sets. Both error bands are normalized to the respective central CT14 NNLO PDFs.

input physics assumption that both $d_{\mathrm{val}}$ and $u_{\mathrm{val}}$ behave as $(1-x)^{a_{2}}$ at $x \rightarrow 1$ with the same value of $a_{2}$ (reflecting expectations from spectator counting rules), but allowing for independent normalizations. The $\bar{u} / \bar{d}$ ratio has also changed as a consequence of the new data and the new parametrization form. The CTEQ-JLab analysis (CJ12) [23] has independently determined the ratio $d / u$ at NLO, and it is compared to that of CT14 in Fig. 2.

$W / Z$ production cross sections at the LHC. Fig. 3 shows central predictions and $90 \%$ C.L. regions for $\left(W^{+}, W^{-}\right),\left(Z, W^{ \pm}\right)$pairs of inclusive cross sections at the LHC 8 and $13 \mathrm{TeV}$. In each subfigure, two elliptical confidence regions are shown, obtained with either CT14 or CT10 NNLO PDFs. These can be used to read off PDF uncertainties and correlations for each pair of cross sections. The plots in Fig. 3 indicate that the PDF uncertainties, at the 90\% C.L., are about 3.9\%, 3.7\%, and $3.7 \%$ for $W^{+}, W^{-}$, and $Z$ boson production at the LHC $13 \mathrm{TeV}$, respectively. The central predictions at $8 \mathrm{TeV}$ are in agreement with the recent CMS measurements [24]. They also show that the electroweak gauge boson cross sections are highly correlated with each other; in fact, much of the uncertainty is driven in this case by the small- $x$ gluon [12]. 

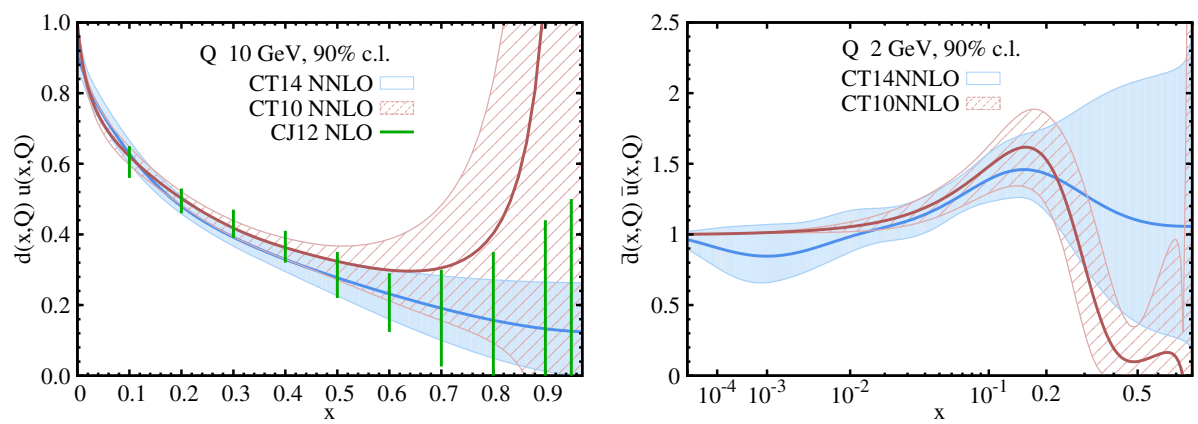

Figure 2. Comparison of $90 \%$ C.L. uncertainties on the ratios $d(x, Q) / u(x, Q)$ for CT14 NNLO (solid blue) and CT10 NNLO (red dashed), and CJ12NLO (green lines) error ensembles and $\bar{d}(x, Q) / \bar{u}(x, Q)$.

Differential cross sections for production of massive vector bosons set important constraints on the flavor composition of the proton, notably on the $u$ and $d$ quarks, anti-quarks and their ratios. In Fig. 4 CT14 NNLO theoretical predictions for lepton charge asymmetries of inclusive $W^{ \pm}$boson production are compared to data measured at the DØ Tevatron Run-2 [16] and ATLAS [13], CMS [14], and $\mathrm{LHCb}$ [15] experiments at the LHC $7 \mathrm{TeV}$. Theoretical predictions are computed using the program ResBos [25-28]. The black data points represent the unshifted central values of the data. The error bars indicate the total (statistical+systematic) experimental error, while the blue band is the CT14 PDF uncertainty evaluated at the $68 \%$ C.L. In total, constraints from the LHC and Tevatron $W / Z$ differential cross sections and asymmetries lead to important changes in the quark sector PDFs. At $x \lesssim 0.02$, more realistic error bands for the $u, \bar{u}, d, \bar{d}$ PDFs are obtained upon including the ATLAS and CMS data sets. At $x>0.1$, the high-luminosity D $\varnothing$ charge asymmetry and other compatible experiments predict a softer behavior of $d(x, Q) / u(x, Q)$ than in CT10W.

Compared to CT10, the new inputs and theoretical advancements of the CT14 fit resulted in a slight increase in the large- $x$ gluon (of order 1\%). The CT14 $g g$ luminosities are compared to those of MMHT2014 [29] and NNPDF3.0 [30] PDFs at $13 \mathrm{TeV}$ in Fig. 5. The parton luminosity is defined as in Ref. [31]. All central values and uncertainty bands agree very well among the three global PDFs, in the $x$ range sensitive to Higgs production.

\section{Conclusions}

A short overview of the CT14 global analysis of proton's PDFs has been given. With rapid advancements in LHC measurements, the focus of the global analysis has shifted toward providing accurate predictions in the wide range of $x$ and $Q$ covered by the LHC data. This development requires longterm multi-prong effort in theoretical, experimental, and statistical areas. On the theory side, a more flexible parametrization has been introduced to better capture variations in the PDF dependence. A series of benchmark tests of NNLO cross sections, carried out in the run-up for the CT14 fit for all key fitted processes, have resulted in better agreement with most experiments and brought accuracy of most predictions to the truly NNLO level. The final CT14 PDFs are presented in the form of 1 central and 56 Hessian eigenvector sets at NLO and NNLO. The 90\% C.L. PDF uncertainties for physical observables can be estimated from these sets using the symmetric [32] or asymmetric [3, 33] master formulae. These PDFs are determined for the central QCD coupling of $\alpha_{s}\left(M_{Z}\right)=0.118$, consistent with the world-average $\alpha_{s}$ value. 

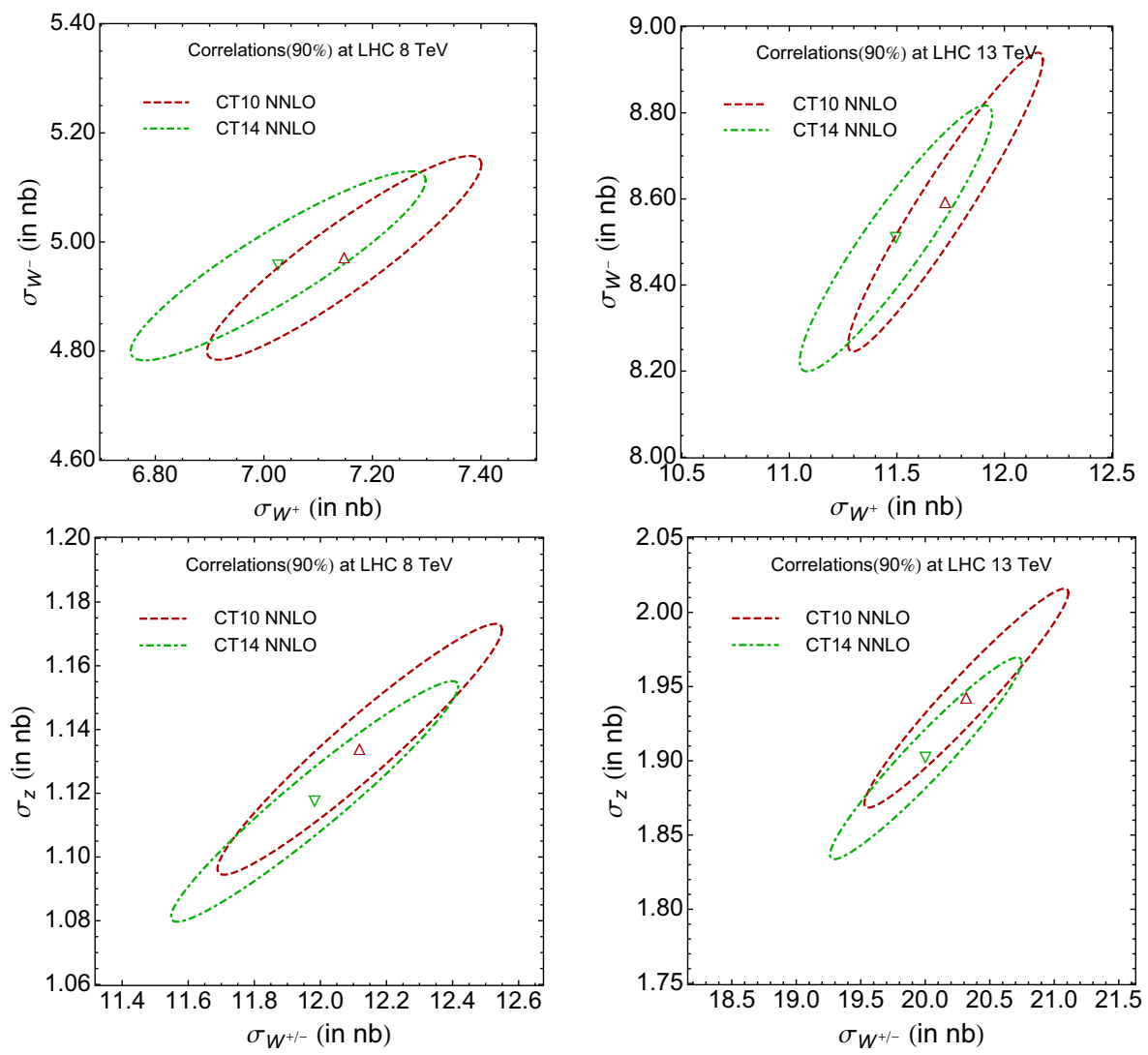

Figure 3. CT14 and CT10 NNLO 90\% C.L. error ellipses for $W^{-}$and $W^{+}$cross sections, at the LHC 8 and 13 $\mathrm{TeV}$. and $Z$ and $W^{ \pm}$.

\section{Acknowledgments.}

M.G. would like to thank the organizers of the ISMD2015 conference for the kind invitation. This work was supported by the STFC (ST/L000431/1) and by the Lancaster-Manchester-Sheffield Consortium for Fundamental Physics under STFC grant ST/L000520/1; by the U.S. DOE Early Career Research Award DE-SC0003870; by the U.S. Department of Energy under Grant No. DE-FG0296ER40969, DE-SC0013681, and DE-AC02-06CH11357; by the U.S. National Science Foundation under Grant No. PHY-0855561 and PHY-1417326; by Lightner-Sams Foundation; and by the National Natural Science Foundation of China under Grant No. 11165014 and 11465018.

\section{References}

[1] G. Aad et al. (ATLAS), Phys. Lett. B716, 1 (2012), 1207.7214

[2] S. Chatrchyan et al. (CMS), Phys. Lett. B716, 30 (2012), 1207.7235

[3] H.-L. Lai, M. Guzzi, J. Huston, Z. Li, P.M. Nadolsky, J. Pumplin, C.-P. Yuan, Phys. Rev. D82, 074024 (2010), 1007.2241 

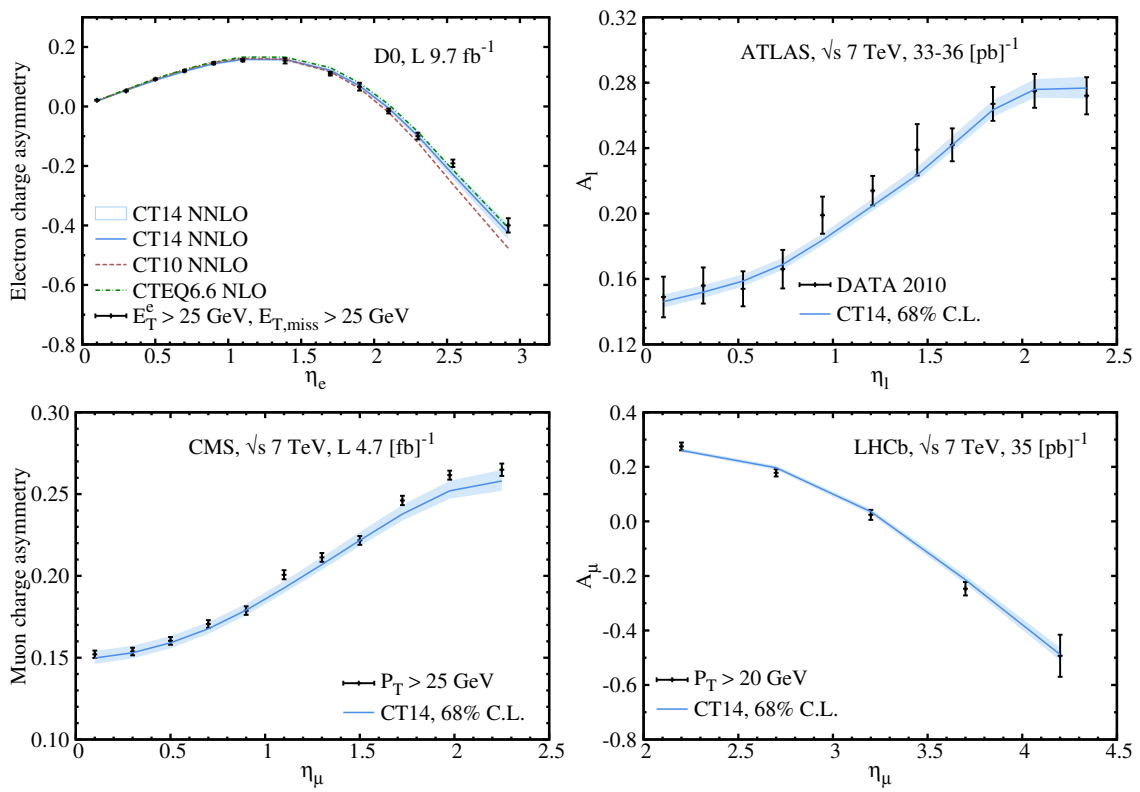

Figure 4. Charge asymmetries as a function of lepton pseudorapidity measured by the DØ, ATLAS, CMS, and LHCb Collaborations, compared to the $68 \%$ C.L. CT14 NNLO uncertainty band.

[4] J. Gao, M. Guzzi, J. Huston, H.L. Lai, Z. Li, P. Nadolsky, J. Pumplin, D. Stump, C.-P. Yuan, Phys. Rev. D89, 033009 (2014), 1302 . 6246

[5] S. Dulat, T.J. Hou, J. Gao, M. Guzzi, J. Huston, P. Nadolsky, J. Pumplin, C. Schmidt, D. Stump, C.-P. Yuan (2015), 1506.07443

[6] https://lhapdf.hepforge.org/

[7] M. Guzzi, P.M. Nadolsky, H.L. Lai, C.-P. Yuan, Phys. Rev. D86, 053005 (2012), 1108 . 5112

[8] M.A.G. Aivazis, J.C. Collins, F.I. Olness, W.-K. Tung, Phys. Rev. D50, 3102 (1994), hep-ph/9312319

[9] J.C. Collins, Phys. Rev. D58, 094002 (1998), hep-ph/9806259

[10] W.-K. Tung, S. Kretzer, C. Schmidt, J. Phys. G28, 983 (2002), hep-ph/0110247

[11] W.-K. Tung, H.L. Lai, A. Belyaev, J. Pumplin, D. Stump, C.-P. Yuan, JHEP 02, 053 (2007), hep-ph/0611254

[12] P.M. Nadolsky, H.L. Lai, Q.H. Cao, J. Huston, J. Pumplin, D. Stump, W.-K. Tung, C.-P. Yuan, Phys. Rev. D78, 013004 (2008), 0802 .0007

[13] G. Aad et al. (ATLAS), Phys. Rev. D85, 072004 (2012), 1109. 5141

[14] S. Chatrchyan et al. (CMS), Phys. Rev. D90, 032004 (2014), 1312.6283

[15] R. Aaij et al. (LHCb), JHEP 06, 058 (2012), 1204 . 1620

[16] V.M. Abazov et al. (D0), Phys. Rev. D91, 032007 (2015), [Erratum: Phys.

Rev.D91,no.7,079901(2015)], 1412.2862

[17] J. Pumplin, D. Stump, R. Brock, D. Casey, J. Huston, J. Kalk, H.L. Lai, W.-K. Tung, Phys. Rev.

D65, 014013 (2001), hep-ph/0101032

[18] G. Watt, R.S. Thorne, JHEP 08, 052 (2012), 1205 . 4024 


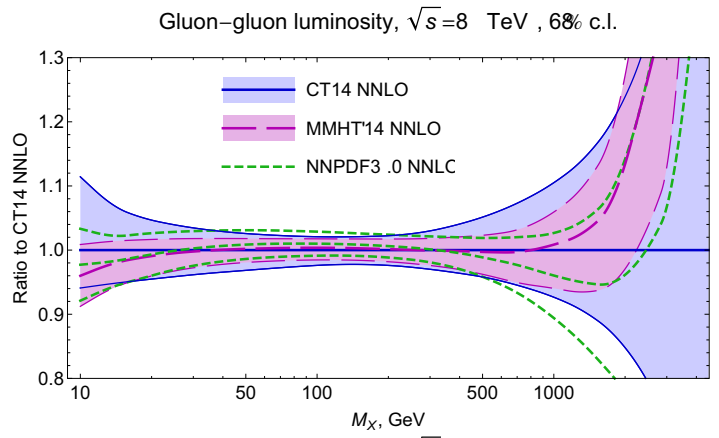

Gluon-gluon luminosity, $\sqrt{s}=13 \mathrm{TeV}, 68 \%$ c.l.

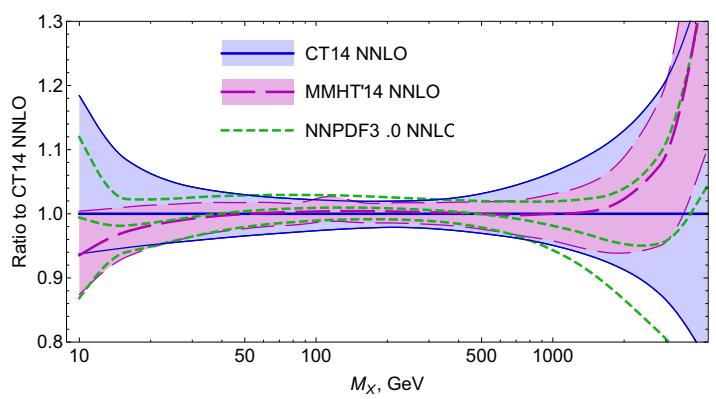

Figure 5. The $g g$ PDF luminosities for CT14, MMHT2014 and NNPDF3.0 PDFs at the LHC with $\sqrt{s}=8$ and $13 \mathrm{TeV}$, with $\alpha_{s}\left(M_{Z}\right)=0.118$.

[19] D. Stump, J. Pumplin, R. Brock, D. Casey, J. Huston, J. Kalk, H.L. Lai, W.-K. Tung, Phys. Rev. D65, 014012 (2001), hep-ph/0101051

[20] K.A. Olive et al. (Particle Data Group), Chin. Phys. C38, 090001 (2014)

[21] S. Alekhin et al. (2011), 1101.0536

[22] V.M. Abazov et al. (D0), Phys. Rev. Lett. 101, 211801 (2008), 0807. 3367

[23] J. Owens, A. Accardi, W. Melnitchouk, Phys.Rev. D87, 094012 (2013), 1212 . 1702

[24] S. Chatrchyan et al. (CMS), Phys. Rev. Lett. 112, 191802 (2014), 1402.0923

[25] C. Balazs, J.w. Qiu, C.-P. Yuan, Phys. Lett. B355, 548 (1995), hep-ph/9505203

[26] C. Balazs, C.-P. Yuan, Phys. Rev. D56, 5558 (1997), hep-ph/9704258

[27] F. Landry, R. Brock, P.M. Nadolsky, C.-P. Yuan, Phys. Rev. D67, 073016 (2003), hep-ph/0212159

[28] M. Guzzi, P.M. Nadolsky, B. Wang, Phys. Rev. D90, 014030 (2014), 1309. 1393

[29] L.A. Harland-Lang, A.D. Martin, P. Motylinski, R.S. Thorne, Eur. Phys. J. C75, 204 (2015), 1412. 3989

[30] R.D. Ball et al. (NNPDF), JHEP 04, 040 (2015), 1410. 8849

[31] J.M. Campbell, J.W. Huston, W.J. Stirling, Rept. Prog. Phys. 70, 89 (2007), hep-ph/0611148

[32] J. Pumplin, D.R. Stump, J. Huston, H.L. Lai, P.M. Nadolsky, W.-K. Tung, JHEP 07, 012 (2002), hep-ph/0201195

[33] P.M. Nadolsky, Z. Sullivan, eConf C010630, P510 (2001), hep-ph/0110378 